\title{
Every orientation of a 4-chromatic graph has a non-bipartite acyclic subgraph
}

\author{
Asaf Shapira* \\ School of Mathematics \\ Tel Aviv University \\ Tel Aviv 69978, Israel \\ asafico@tau.ac.il
}

Submitted: Sep 14, 2021; Accepted: Dec 27, 2021; Published: Jan 28, 2022

(C) The author. Released under the CC BY-ND license (International 4.0).

\begin{abstract}
Let $f(n)$ denote the smallest integer such that every directed graph with chromatic number larger than $f(n)$ contains an acyclic subgraph with chromatic number larger than $n$. The problem of bounding this function was introduced by AddarioBerry et al., who noted that $f(n) \leqslant n^{2}$. The only improvement over this bound was obtained by Nassar and Yuster, who proved that $f(2)=3$ using a deep theorem of Thomassen. Yuster asked if this result can be proved using elementary methods. In this short note we provide such a proof.
\end{abstract}

Mathematics Subject Classifications: 05C15, 05C20

\section{Introduction}

The relation between the chromatic number of a graph and properties of orientations of its edges have long been investigated. For the sake of brevity, we refer the reader to [4] for a general survey on this topic, and to the discussions in $[3,6]$, which are more closely related to our investigation here.

We consider the following problem introduced by Addario-Berry, Havet, Sales, Reed and Thomassé; given an integer $n$, what is the smallest integer $f(n)$ so that if $G$ has chromatic number more than $f(n)$ then in every orientation of $G$ 's edges, one can find an acyclic subgraph of chromatic number more than $n$. The best known general upper bound for this function is $f(n) \leqslant n^{2}$. This follows from taking any oriented version of $G$, splitting it into two acyclic subgraphs, denoted $G_{1}, G_{2}$, and applying the well known

\footnotetext{
*Supported in part by ISF Grant 1028/16, ERC Consolidator Grant 863438 and NSF-BSF Grant 20196.
} 
fact that the chromatic number of $G$ is at most the product of the chromatic numbers of $G_{1}, G_{2}$. The only known improvement over this general bound was obtained by Nassar and Yuster [6] who proved that $f(2)=3$, by establishing the following.

Theorem 1 (Nassar-Yuster [6]). Suppose $G$ is a graph of chromatic number 4. Then every orientation of its edges contains an acyclic odd cycle.

The proof in [6] relied on a deep theorem of Thomassen [7], which confirmed a conjecture of Toft [8]. Yuster [9] asked if one can prove Theorem 1 using elementary methods. In this short paper we give such a proof. The main idea is to take advantage of properties of 4-critical graphs.

\section{An elementary proof of Theorem 1}

We may and will assume that $G$ is 4-critical, that is, that the removal of every edge of $G$ reduces its chromatic number. This will allow us to use important properties of 4-critical graphs. We proceed by induction on $|V(G)|$, with the base case being $K_{4}$. It is easy to see that every orientation of $K_{4}$ contains an acyclic $K_{3}$ (in fact, two) so the base case holds. We now proceed with the induction step. We consider separately the case where $G$ is 3 -connected (in which case we will not need induction) and the case where it has a separating pair of vertices.

Assume first that $G$ is 3-connected, and let $C$ be a shortest odd cycle in $G$. Since $C$ must be induced and $G$ has chromatic number 4 , there must be a vertex $v \notin C$. Since $G$ is assumed to be 3 -connected, there are 3 vertex disjoint paths connecting $v$ to $C$. Let $P, Q, R$ denote these paths, and $p, q, r$ denote their meeting points with $C$, see Figure 1a. If $C$ is acyclic we are done, so suppose wlog that $C$ is oriented as in Figure 1a. Clearly not all three paths $P \cup Q, P \cup R$ and $Q \cup R$ can be directed paths, as they all intersect internally in the vertex $v$. Assume wlog that $P \cup Q$ is not directed. Then, since $|C|$ is odd, one of the cycles $P \cup Q \cup P_{p q}$ or $P \cup Q \cup P_{r p} \cup P_{q r}$ is an acyclic odd cycle.

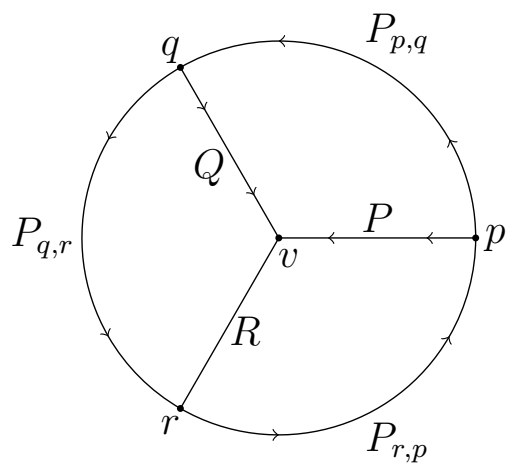

(a) $G$ is 3-connected

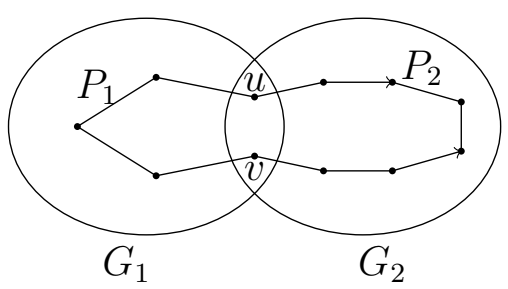

(b) $G$ is not 3-connected

Figure 1: The two cases considered in the proof 
Suppose now that $G$ is not 3 connected, that is, it has a pair of vertices $u, v$ whose removal breaks it into at least two (non-empty) connected components. In what follows, if $(u, v) \notin E(G)$ then we use $G+(u, v)$ to denote the graph obtained by adding the edge $(u, v)$ to $G$. We use $G /\{u, v\}$ to denote the graph obtained from $G$ by contracting $u, v$, that is, the graph obtained by replacing $u, v$ with a new vertex and connecting it to all the vertices that were connected to either ${ }^{1} v$ or $u$. We will need the following well known result of Dirac [2], see also Problem 9.22 in [5] for a short proof.

Lemma 2 (Dirac [2]). Let $k \geqslant 4$ be an integer, let $G$ be a $k$-critical graph, and let $u, v \in V(G)$ be such that $G \backslash\{u, v\}$ (the graph obtained from $G$ by deleting the vertices $u, v)$ is disconnected. Then:

1. $u \neq v$, that is, $G$ is 2-connected;

2. $(u, v) \notin E(G)$;

3. $G \backslash\{u, v\}$ has exactly two components;

4. There are unique proper induced subgraphs $G_{1}, G_{2}$ of $G$ such that $G=G_{1} \cup G_{2}$, $V\left(G_{1}\right) \cap V\left(G_{2}\right)=\{u, v\}$, and the graphs $G_{1} \backslash\{u, v\}$ and $G_{2} \backslash\{u, v\}$ are the two components of $G \backslash\{u, v\}$. Also, $u, v$ have no common neighbor in $G_{2}$, and $G_{1}+(u, v)$ and $G_{2} /\{u, v\}$ are $k$-critical.

By induction and Lemma 2, the graph $G_{2} /\{u, v\}$ has an acyclic odd cycle $C_{2}$. If $C_{2}$ does not contain the vertex $w$ that resulted from contracting $\{u, v\}$, it is also a cycle in $G$ and we are done. Also, if the two neighbors of $w$ on $C_{2}$ are both neighbors of $v$ or both neighbors of $u$, then we can again conclude that $C_{2}$ is also an acyclic odd cycle in $G$. So assume one neighbor of $w$ is a neighbor of $v$ and one is a neighbor of $u$. Then we may infer that in $G$ we have a path $P_{2}$ connecting $u$ and $v$, so that $|P|$ is even and $P$ is not directed from $u$ to $v$ or from $v$ to $u$. See Figure $1 \mathrm{~b}$.

By induction and Lemma 2, the graph $G_{1}+(u, v)$ has an acyclic odd cycle $C_{1}$ (no matter how we orient the edge $(u, v))$. If $C_{1}$ does not use the edge $(u, v)$, it is also an acyclic odd cycle in $G$ and we are done. Suppose then that it does, implying that $G$ contains a path $P_{1}$ connecting $u$ to $v$ with $\left|P_{1}\right|$ odd. Then item (4) in Lemma 2 guarantees that $\left|P_{1} \cup P_{2}\right|=\left|P_{1}\right|+\left|P_{2}\right|-2$ so $P_{1} \cup P_{2}$ is an odd cycle. The assertion at the end of the previous paragraph guarantees that it is acyclic. This completes the proof of Theorem 1 .

\section{Acknowledgements}

I would like to thank Lior Gishboliner for helpful remarks and for producing Figure 1.

\footnotetext{
${ }^{1}$ We will only apply this operation when $u, v$ are not connected and have no common neighbor, so this operation will not create loops or parallel edges.
} 


\section{References}

[1] L. Addario-Berry, F. Havet, C. L. Sales, B. Reed, and S. Thomassé. Oriented trees in digraphs. Discrete Math., 313:967-974, 2013.

[2] G. A. Dirac. On the structure of 5- and 6-chromatic abstract graphs. J. Reine Angew. Math. 214/215:43-52, 1964.

[3] J. Fox, M. Kwan and B. Sudakov. Acyclic subgraphs of tournaments with high chromatic number. Bull. London Math. Soc. 53:619-630, 2021.

[4] F. Havet, Orientations and colouring of graphs. Lecture notes of SGT 2013, Oleron, France.

[5] L. Lovász. Combinatorial Problems and Exercises. North Holland, Amsterdam, 1979, Problem 10.29.

[6] S. Nassar and R. Yuster. Acyclic subgraphs with high chromatic number. European J. Combin. 75:11-18, 2019.

[7] C. Thomassen. Totally odd $K_{4}$-subdivisions in 4-chromatic graphs. Combinatorica 21:417-443, 2001.

[8] B. Toft, Problem 11, In Recent Advances in Graph Theory, pages 543-544, Academia Praha, 1975.

[9] R. Yuster, Lecture at Tel Aviv University Combinatorics Seminar, 2019. 